\title{
Coordinated voltage regulation of hybrid AC/DC medium voltage distribution networks
}

\author{
Lu ZHANG ${ }^{1}$, Ying CHEN $^{1}$, Chen SHEN $^{1}$ (i), Wei TANG ${ }^{2}$, \\ Jun LIANG ${ }^{3}$
}

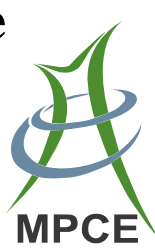

\begin{abstract}
In a hybrid AC/DC medium voltage distribution network, distributed generations (DGs), energy storage systems (ESSs), and the voltage source converters (VSCs) between $\mathrm{AC}$ and $\mathrm{DC}$ lines, have the ability to regulate node voltages in real-time. However, the voltage regulation abilities of above devices are limited by their ratings. And the voltage regulation efficiencies of these devices are also different. Besides, due to high $r / x$ ratio, node voltages are influenced by both real and reactive power. In order to achieve the coordinated voltage regulation in a hybrid $\mathrm{AC} /$ DC distribution network, a priority-based real-time control strategy is proposed based on the voltage control effect of real and reactive power adjustment. The equivalence of real and reactive power adjustment on voltage control is
\end{abstract}

CrossCheck date: 1 August 2017

Received: 26 February 2017/Accepted: 1 August 2017/Published online: 11 October 2017

(C) The Author(s) 2017. This article is an open access publication

$\triangle$ Chen SHEN

shenchen@mail.tsinghua.edu.cn

Lu ZHANG

luzhang0210@gmail.com

Ying CHEN

chen_ying@tsinghua.edu.cn

Wei TANG

wei_tang@cau.edu.cn

Jun LIANG

LiangJ1@ cardiff.ac.uk

1 Department of Electrical Engineering, Tsinghua University, Beijing 100084, China

2 College of Information and Electrical Engineering, China Agricultural University, Beijing 100083, China

3 School of Engineering, Cardiff University, Cardiff CF24 3AA, UK considered in control area partition optimization, in which regulation efficiency and capability are taken as objectives. In order to accommodate more DGs, the coordination of controllable devices is achieved according to voltage sensitivities. Simulations studies are performed to verify the proposed method.

Keywords Hybrid AC/DC, Distribution network, Voltage control, Partition, Power system

\section{Introduction}

Medium voltage distribution networks with high penetrations of distributed generations (DGs) are facing the challenge of nodal voltage regulations. Generally, in traditional AC distribution networks, voltage profiles are maintained through controlling on-load-tap-change transformers [1], shunt capacitors [2] and circuit breakers [3]. In a hybrid AC/DC distribution network, coordinated regulations of converter-based controllable devices are highly demanded for voltage control.

To mitigate probabilistic voltage violations, day-ahead dispatching methods have been presented in the literature $[4,5]$. However, if unexpected fast variation of DG outputs and loads happen, day-ahead optimal schedules may fail to retain voltage profiles of all buses. In these circumstances, real time regulations of controllable devices should be applied to remain the voltage of important buses, which consequently also reduce undesirable DG curtailments and load shedding.

DGs and energy storage systems (ESSs) have been investigated to achieve real-time voltage regulation in distribution networks [6-12]. Moreover, in order to increase transfer capacities and DG accommodations, some 
AC lines can be converted into DC ones and forms a hybrid AC/DC distribution network by connecting existing AC lines through voltage source converters (VSCs) [13]. Then, real time regulations could be improved through utilizing flexible and fast control ability of VSCs. Ideally, controllable devices including ESSs, DGs, and the VSCs connecting AC and DC lines should be considered together to generate optimal real-time regulation strategies for the hybrid AC/DC distribution networks.

In distribution networks, nodal voltages are influenced by both real and reactive power flows due to high $r / x$ ratio of lines [14]. Aforementioned controllable devices usually are connected to the distribution network through converter based interfaces. They have the ability of controlling their real power and reactive power independently. Thus, they can impact the system voltages through adjusting either output real power or reactive power. However, the power ratings of these converter-based devices are limited; their real and reactive power outputs are not fully decoupled. Therefore, during real time regulations, real and reactive power controls of various devices should be coordinated carefully.

Various methods of real-time regulations related to DGs and ESSs have been proposed in literatures. Reference [7] proposed a real-time voltage control method considering coordination of multiple battery ESSs. In [8], real-time market transactions, online dispatch of DGs and load curtailments are deployed in order to minimize expected operating costs. The reactive power capability of inverters and the technical requirement of DGs are analyzed in [9]. An Optimal control management of ESSs is proposed to mitigate the fluctuation and intermittence of renewable generations in [10]. The correlation between DGs is considered in a hybrid energy storage system in [11]. Reference [12] establishes an optimal economic operation mode for community microgrid incorporating temperature controlling devices with DGs and ESS. In [15], a centralized real-time control system is proposed to optimize the reactive power of DGs. Reference [16] leverages DC grid interconnections to enhance transfer capacities, and regulate voltages at AC feeder terminals. Reference [17] presents a centralized two-stage stochastic dispatch scheme, in which the day-ahead dispatch orders for controllable DG units are determined in the first stage, while appropriate corrective decisions are determined in the second stage.

In order to achieve an efficient local control, some papers proposed to use partition methods for voltage regulation [18-20]. A graph is introduced to represent a distribution network, in which controllable devices are connected to different nodes. A graph partition method is to divide the graph into a given number of sub-graphs, named control areas. Then, voltage regulation scheme can be designed and implemented separately for each control area. In [18], an analytical partition method based on capacitor reactive power is presented. Reference [19] divided the power system into regions based on a graph partition method and investigated a secondary voltage control for each region to prevent the propagation of disturbances. Reference [20] developed an approach for voltage and reactive power control in order to deal with the fluctuations caused by intermittent changes of renewable resources. However, in order to achieve local compensation of reactive power in $\mathrm{AC}$ system, existing references only considered reactive power, which does not exist in DC lines. Moreover, the control capability of both real and reactive power needs to be considered in a hybrid AC/DC medium voltage distribution network due to its flexible control capability and high $r / x$.

This paper proposes a coordinated real-time voltage regulation method for hybrid AC/DC medium distribution networks. This method makes full use of converter-based controllable devices, such as DGs, ESSs, and the VSCs between AC and DC lines. To eliminating possible conflicting controls, an optimal partition method is developed, which helps to determine suitable devices to retain node voltages against various uncertainties. This partition method commits to multiple objectives, in which both efficiency and capability of real and reactive power regulations are considered. Through a sensitivity analysis method, real and reactive power outputs of controllable devices can be adjusted to retain nodal voltages in real time operations. As power balances of both AC and DC grids are considered, these adjustments tend to be coordinated and help to minimize DG curtailments and load shedding. Finally, based on resultant network partition, priorities of control actions can be evaluated, according to which corresponding devices are controlled with special and temporal coordination.

\section{Problem description}

Figure 1 illustrates the structure of a hybrid AC/DC distribution network with DGs and ESSs, where VSC1 controls the DC voltage and VSC2 controls real and reactive power to $\mathrm{AC}$ lines. It is assumed that: (1) the voltage of the secondary sides of distribution transformers is fixed and cannot be regulated in real time; (2) power flow of the distribution grid keeps changing due to fluctuations of both DG outputs and load dmands; (3) voltage violations could happen as the day-ahead optimal scheduling is based on probabilistic forecasts of DG outputs and load demands; (4) For ESSs, the state of charge (SOC) and charging/discharging times are considered in the day-ahead optimal scheduling. Thus, a real time control method should be developed, which adjusts controllable devices in every 


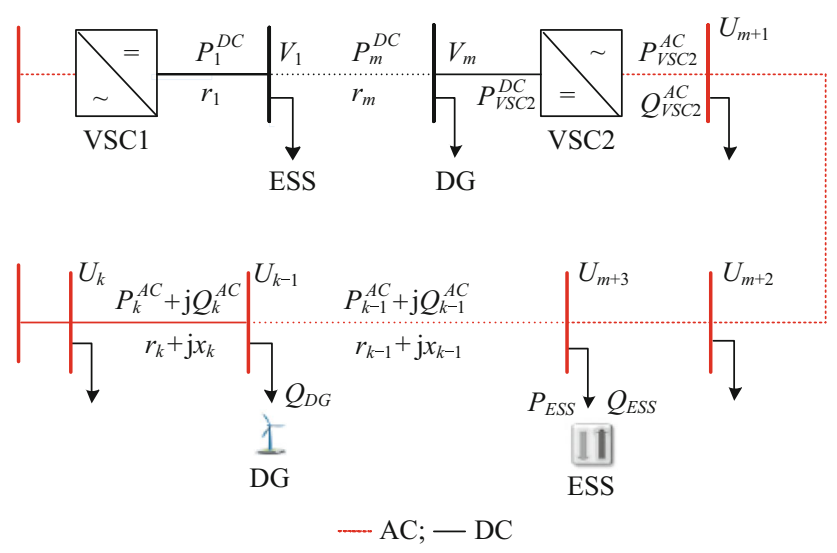

Fig. 1 Structure of a hybrid AC/DC distribution network

short time interval using latest measurements. A central controller is assumed to regulate controllable devices by using wireless wide-area communication.

It should be noted that in a hybrid AC/DC distribution system, both real and reactive power flows can be adjusted to improve nodal voltage profiles. Thus, VSCs, ESSs and DGs can be considered as controllable devices for the aforementioned real-time voltage control. However, constraints of these controllable devices such as ratings of the converters must be respected. The reactive power output capacity of a DG is represented by:

$Q_{D G}=\sqrt{S_{D G}^{2}-P_{D G}^{2}}$

where $S_{D G}$ is the rated capacity of a DG; $P_{D G}$ is the real power output of the DG.

For VSC2 and ESSs, real power outputs $\left(P_{V S C 2}^{A C}, P_{E S S}\right)$ and reactive power outputs $\left(Q_{V S C 2}^{A C}, Q_{E S S}\right)$ satisfy:

$$
\left\{\begin{array}{l}
P_{V S C 2}^{A C}+Q_{V S C 2}^{A C}{ }^{2}=S_{V S C 2}{ }^{2} \\
P_{E S S}{ }^{2}+Q_{E S S}{ }^{2}=S_{E S S}{ }^{2}
\end{array}\right.
$$

where $S_{V S C 2}$ is the capacity of VSC2; $S_{E S S}$ is the maximum available capacity of an ESS according to its SOC.

There are two stages to achieve the proposed voltage regulation for hybrid AC/DC distribution networks. In the first stage, an offline partition method is conducted to divide a distribution network into control areas, and the priority of controllable devices is determined. In the second stage, a central controller is assumed to measure node voltages and regulate the outputs of controllable devices using a linearized voltage-sensitivity approach. A top level control strategy diagram is shown in Fig. 2.

In particular, control areas are carefully defined with considerations of voltage regulation capability and efficiency of all controllable devices. Both real and reactive power outputs of VSCs, ESSs and DGs are utilized for nodal voltage regulation. Thus, to select suitable controllable

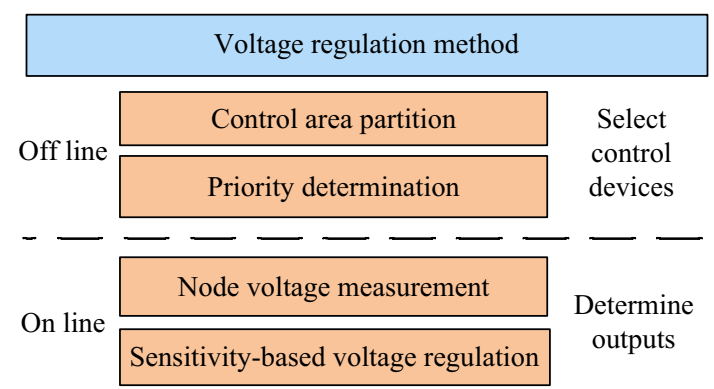

Fig. 2 A top level control strategy diagram

devices for each important bus, a multi-objective optimal partition is formulated, and a priority-based coordination strategy is determined for the controllable devices in each control area.

Then, the coordination of real and reactive power controls of different controllable devices should be conducted. Power exchanges through the VSC2 $\left(P_{V S C 2}^{A C}\right.$ and $\left.Q_{V S C 2}^{A C}\right)$ can be adjusted flexibly, which helps to control power flow of the AC grid. Meanwhile, ESSs ( $P_{E S S}$ and $\left.Q_{E S S}\right)$ and DGs $\left(P_{D G}, Q_{D G}\right)$ can also be used to regulate node voltages, whose power outputs reshape the power flow of a control area directly. Adjustments of power outputs of these controllable devices should be determined regarding temporal sequence of their actions. Therefore, a voltage-sensitivity approach is used to determine the adjustments, based on which unnecessary curtailments of DGs could be avoided, while keeping nodal voltage acceptable.

\section{Partition method of hybrid AC/DC distribution network considering both real and reactive power}

\subsection{Equivalent of real power to reactive power for node voltage regulation}

Quantitative relationships between node voltages and injected powers can be expressed as:

$\Delta V_{i}=\Delta P_{j} S_{v p}(i, j)+\Delta Q_{j} S_{v q}(i, j)$

where $\Delta V_{i}$ is the voltage variation of the node $i ; S_{v p}$ and $S_{v q}$ are the inverse Jacobian matrixes in Newton-Raphson algorithm [21]; $\Delta P_{j}$ and $\Delta Q_{j}$ are variations of injected real and reactive power of node $j$.

For DC lines, $\Delta Q_{j}$ is zero because there is no reactive power flowing in DC lines, and only real power can be used to regulate node voltages.

Equation (3) can be represented as:

$\Delta V_{i}=\left(\Delta P_{j} \frac{S_{v p}(i, j)}{S_{v q}(i, j)}+\Delta Q_{j}\right) S_{v q}(i, j)$ 
Thus, the influence of real power on node voltage can be represented as that of reactive power in equivalence according to (4).

The regulation efficiency and capability of both real and reactive powers at node $j$ to the voltage of node $i$ can be represented by $S_{v q}(i, j)$ and $\Delta P_{j} \frac{S_{v p}(i, j)}{S_{v q}(i, j)}+\Delta Q_{j}$.

\subsection{Multi-objective partition model of hybrid AC/ DC distribution network}

In order to achieve the coordination between $P_{V S C 2}^{A C}$, $Q_{V S C 2}^{A C}, P_{E S S}, Q_{E S S}$ and $Q_{D G}$, a multi-objective partition method of hybrid AC/DC distribution network is proposed aiming at choosing a most efficient regulation device for each node. In the optimization, the connectivity of each partition should be ensured, so the number of branches is optimized as variables.

The partition objective of hybrid AC/DC distribution network is shown in (5), in which the regulation efficiency and capability of both real and reactive power are considered. The minimization of electrical distances between nodes is used to present the regulation efficiency, while the sum of squares of deviations (SSD) of real and reactive power reserve is used to present the regulation capability.

$\left\{\begin{array}{l}f_{1}=\min \prod_{i=1}^{N_{p a r}} D_{i, \max } \\ f_{2}=\min \sum_{i=1}^{N_{p a r}}\left(R_{i}-\bar{R}\right)^{2}\end{array}\right.$

where $D_{i, \max }$ is the maximum electrical distance between nodes in partition $i ; R_{i}$ is the power reserve of real and reactive power in partition $i ; \bar{R}$ is the average power reserve of hybrid AC/DC distribution network. $N_{\text {par }}$ is the number of partition.

The number of partition is assumed to be the total number of ESSs and VSC2 in a hybrid AC/DC medium voltage distribution network. Because only an ESS and VSC2 are able to regulate real power (the real power of DGs is determined by renewable resources) and it is assumed that the curtailment of DGs is only acceptable when voltage violations happen, each partition must has an ESS or VSC2 to regulate the voltage. In order to ensure that there is an ESS or VSC2 in each partition, a constraint shown in (6) is included in the optimization.

$P_{s}^{i}>0$

where $P_{s}^{i}$ is the available real power control capacity offered by controllable devices in partition $i$.

The electrical distance between node $k$ and node $n$ in Fig. 1 can be calculated as [22]:
$D_{k n}=-\lg \left(\alpha_{k n} \alpha_{n k}\right)$

$\alpha_{k n}=\frac{S_{v q}(k, n)}{S_{v q}(n, n)}$

where $D_{k n}$ is the electrical distance between node $k$ and node $n$.

The power reserve in a partition $R_{i}$ can be calculated according to (9), which considers both the real and reactive power capabilities of ESS and VSC2 based on (4):

$R_{i}=\frac{\sum_{s=1}^{n} P_{C D, s}^{i} \sum_{l=1}^{m} S_{v p}(l, s)+\sum_{s=1}^{n} Q_{C D, s}^{i} \sum_{l=1}^{m} S_{v q}(l, s)}{\sum_{j=1}^{m}\left(P_{L}^{j}+Q_{L}^{j}\right) \sum_{l=1}^{m} S_{v q}(l, s)}$

where $n$ is the number of controllable devices in partition $i$; $m$ is the number of nodes in partition $i ; P_{L}^{j}$ and $Q_{L}^{j}$ are real and reactive power load at node $j$ in partition $i ; P_{C D, s}^{i}$ and $Q_{C D, s}^{i}$ are real and reactive power capabilities of controllable devices in partition $i$.

In order to consider the maximum regulation capabilities, $P_{C D, s}^{i}$ and $Q_{C D, s}^{i}$ are calculated based on the maximum voltage deviation $\Delta V$ that can be regulated.For DGs and ESSs, $P_{C D, s}^{i}$ and $Q_{C D, s}^{i}$ can be calculated by (10).

$$
\left\{\begin{array}{l}
\max \sum_{l=1}^{m}\left|\Delta V_{l}\right| \\
\sum_{l=1}^{m}\left|\Delta V_{l}\right|=\sum_{l=1}^{m} \sum_{l=1}^{m} S_{v p}(l, s)\left|P_{C D, s}^{i}\right|+\sum_{l=1}^{m} \sum_{l=1}^{m} S_{v q}(l, s)\left|Q_{C D, s}^{i}\right| \\
P_{C D, s}^{i}{ }^{2}+Q_{C D, s}^{i}{ }^{2} \leq S_{C D}^{i}{ }^{2}
\end{array}\right.
$$

where $S_{C D}^{i}$ is rated capacity of DG or ESS in partition $i$. $P_{C D, s}^{i}$ and $Q_{C D, s}^{i}$ can be obtained from (11).

$$
\left\{\begin{array}{l}
P_{C D}^{i}=\frac{\sum_{l=1}^{m} S_{v p}(l, s) S_{C D}^{i}}{\sqrt{\sum_{l=1}^{m} S_{v p}(l, s)^{2}+\sum_{l=1}^{m} S_{v q}(l, s)^{2}}} \\
Q_{C D}^{i}=\frac{\sum_{l=1}^{m} S_{v q}(l, s) S_{C D}^{i}}{\sqrt{\sum_{l=1}^{m} S_{v p}(l, s)^{2}+\sum_{l=1}^{m} S_{v q}(l, s)^{2}}}
\end{array}\right.
$$

For VSC2, the voltage deviations of both AC and DC sides must be considered at the same time, and the power at the DC side is $P_{V S C 2, s}^{i D C}=P_{V S C 2, s}^{i A C}+P_{V S C 2, S}^{L O S S}$. So $P_{V S C 2, s}^{i A C}$, $Q_{V S C 2, s}^{i A C}$ can be calculated through (12). 


$$
\left\{\begin{array}{l}
\max \sum_{l=1}^{m}\left(\left|\Delta V_{l A C}\right|+\left|\Delta V_{l D C}\right|\right) \\
\sum_{l=1}^{m}\left|\Delta V_{l A C}\right|=\sum_{l=1}^{m} S_{v p}^{A C}(l, s)\left|P_{V S C 2, s}^{i A C}\right|+\sum_{l=1}^{m} S_{v q}^{A C}(l, s)\left|Q_{V S C, s}^{i A C}\right| \\
\sum_{l=1}^{m}\left|\Delta V_{l D C}\right|=\sum_{l=1}^{m} S_{v p}^{D C}(l, s)\left|P_{V S C 2, s}^{i D C}\right| \\
P_{V S C 2, s}^{i}{ }^{2}+Q_{V S C 2, s}^{i} 2^{2} \leq S_{V S C 2}^{i}{ }^{2} \\
P_{V S C 2, s}^{i D C}=P_{V S C 2, s}^{i A C}+P_{V S C 2, S}^{L O S S}
\end{array}\right.
$$

where $\left|\Delta V_{l A C}\right|$ and $\left|\Delta V_{l D C}\right|$ are the maximum node voltages deviation that can be regulated in $\mathrm{AC}$ and $\mathrm{DC}$ lines, respectively; $P_{V S C 2, S}^{L O S S}$ is the loss of VSC2, which is assumed to be a fixed factor in this paper. $P_{V S C 2, s}^{i A C}$ and $Q_{V S C 2, s}^{i A C}$ can be obtained by:

$$
\left\{\begin{array}{l}
P_{V S C 2, S}^{i A C}=\frac{\left(\sum_{l=1}^{m} S_{v p}^{A C}(l, s)+\sum_{l=1}^{n} S_{v p}^{D C}(l, s)\right) S_{V S C 2}^{i}}{\sqrt{\left(\sum_{l=1}^{m} S_{v p}^{A C}(l, s)+\sum_{l=1}^{n} S_{v p}^{D C}(l, s)\right)^{2}+\sum_{l=1}^{m} S_{v q}(l, s)^{2}}} \\
Q_{V S C 2, S}^{i A C}=\frac{\left(\sum_{l=1}^{m} S_{v p}^{A C}(l, s)+\sum_{l=1}^{n} S_{v p}^{D C}(l, s)\right) S_{V S C 2}^{i}}{\sqrt{\left(\sum_{l=1}^{m} S_{v p}^{A C}(l, s)+\sum_{l=1}^{n} S_{v p}^{D C}(l, s)\right)^{2}+\sum_{l=1}^{m} S_{v q}(l, s)^{2}}}
\end{array}\right.
$$

where $m$ and $n$ are node number of $\mathrm{AC}$ and $\mathrm{DC}$ lines respectively.

\subsection{Optimization algorithm of multi-objective partition model}

A Pareto-based NSGA-II algorithm [23] is used to solve the proposed multi-objective problem, and the set pair analysis (SPA) theory is used for decision-making [24].

In order to ensure the connectivity of partition result, each branch is represented as a gene, $g_{i}$, using binary encoding, as shown in (14).

$g=\left(g_{1}, g_{2}, \cdots, g_{n}\right)$

where $n$ is the number of branches in hybrid AC/DC distribution network.

If the two nodes of branch $i$ are within the same partition $g_{i}$ is 0 , or $g_{i}$ is 1 .

The elitist strategy is used in the algorithm in order to improve the convergence, and keep Pareto-optimal results to next generation.

A flowchart of the proposed multi-objective partition method of hybrid AC/DC distribution network based on the coordination of real and reactive power is shown in Fig. 3 .

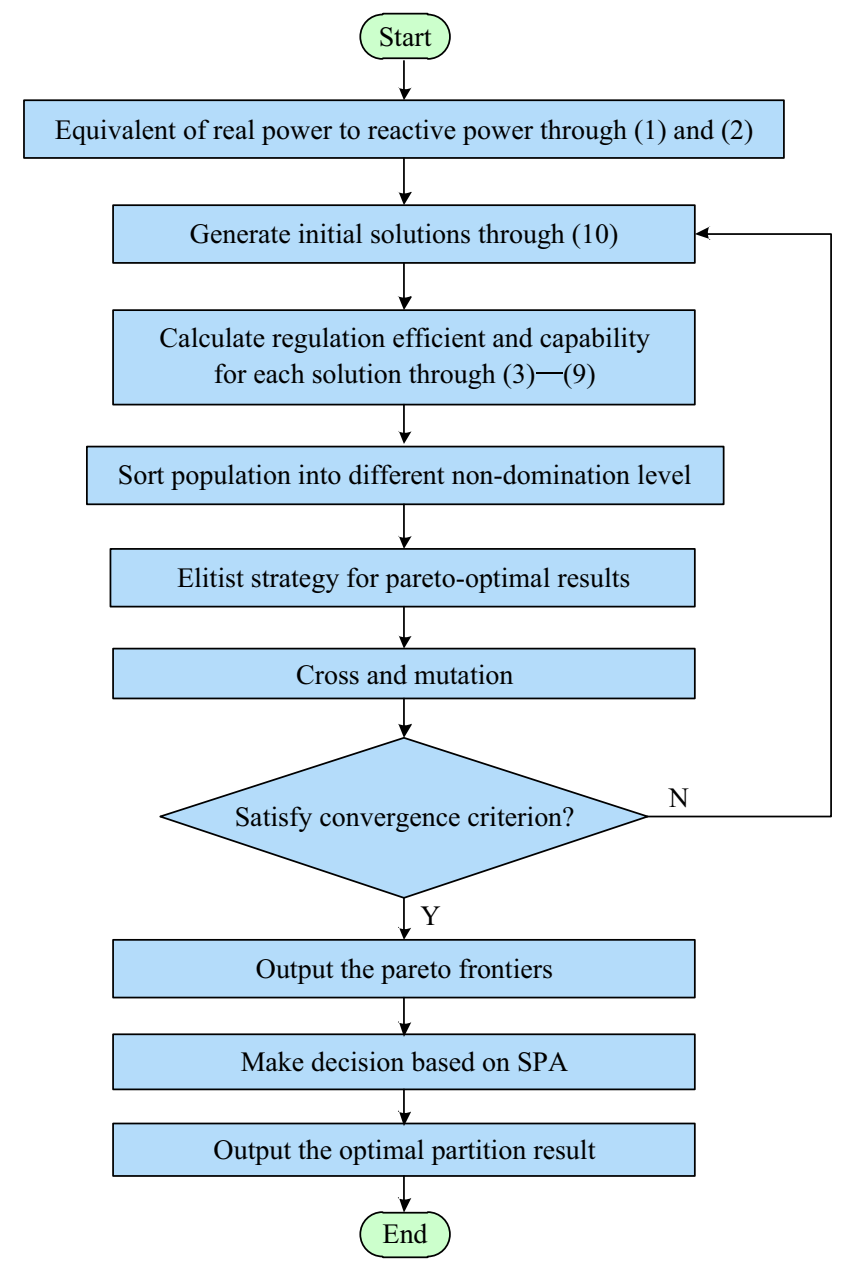

Fig. 3 Flowchart of the proposed multi-objective partition method for hybrid AC/DC distribution network

\section{Coordinated real time voltage control of hybrid AC/DC distribution networks}

In order to regulate node voltages efficiently and flexibly, a novel coordinated voltage control strategy for hybrid AC/DC medium voltage distribution networks is proposed considering the controllable devices of VSC2 $\left(P_{V S C 2}^{A C}\right.$ and $\left.Q_{V S C 2}^{A C}\right)$, ESS $\left(P_{E S S}\right.$ and $\left.Q_{E S S}\right)$, and DG $\left(P_{D G}\right.$ and $\left.Q_{D G}\right)$. A priority-based coordination is carried out according to partition results, considering the regulation costs of each device.

\subsection{Voltage-sensitivity approach}

The coordinated voltage control strategy is triggered if a node voltage is above 1.05 p.u. or below 0.95 p.u.. The real-time voltage control will only switch back to the dayahead optimal scheme if all node voltages are within [0.97, 1.03]. Hence, a linearized regulation can be formulated for 
remaining node voltages within a security operation range.

A sensitivity of a node voltage to the real and reactive power of controllable devices can be obtained through (15) and (16) [25].

$S_{P}=\frac{\partial V_{i}}{\partial P_{s}}=-\frac{R_{i, s}}{V_{n}}$

$S_{Q}=\frac{\partial V_{i}}{\partial Q_{s}}=-\frac{X_{i, s}}{V_{n}}$

where $V_{n}$ is rated voltage; $R_{i, s}$ and $X_{i, s}$ are resistance and reactance between node $i$ and controllable devices. $S_{Q}$ is only evaluated for AC lines.

Thus, the real and reactive power adjustment of controllable devices can be achieved as:

$$
\begin{aligned}
& {[\Delta P]=[\Delta V] /\left[S_{P}\right]} \\
& {[\Delta Q]=[\Delta V] /\left[S_{Q}\right]}
\end{aligned}
$$

where $[\Delta V]$ is the deviation between the measured voltage and a lower or an upper limit, which is 0.97 p.u. for the under-voltage and 1.03 p.u. for the over-voltage cases respectively. These adjustments affect all node voltages which are represented in (19).

$$
\begin{aligned}
{\left[\begin{array}{c}
\Delta V_{1} \\
\vdots \\
\Delta V_{m} \\
\Delta V_{m+1} \\
\vdots \\
\Delta V_{k}
\end{array}\right]=} & {\left[\begin{array}{cccccc}
S_{P}^{1} & \cdots & S_{P}^{1} & S_{P}^{1} & \ldots & S_{P}^{1} \\
S_{P}^{1} & \ddots & \vdots & \vdots & \ddots & \vdots \\
S_{P}^{1} & \cdots & S_{P}^{m} & S_{P}^{m} & \ldots & S_{P}^{m} \\
S_{P}^{1} & \cdots & S_{P}^{m} & S_{P}^{m+1} & \cdots & S_{P}^{m+1} \\
S_{P}^{1} & \ddots & \vdots & \vdots & \ddots & \vdots \\
S_{P}^{1} & \cdots & S_{P}^{m} & S_{P}^{m+1} & \cdots & S_{P}^{k}
\end{array}\right] \cdot\left[\begin{array}{l}
\Delta P_{1} \\
\vdots \\
\Delta P_{m} \\
\Delta P_{m+1} \\
\vdots \\
\Delta P_{k}
\end{array}\right] } \\
& +\left[\begin{array}{cccc}
0 & \ldots & 0 \\
\vdots & \ddots & \vdots \\
0 & \ldots & 0 \\
S_{Q}^{m+1} & \ldots & S_{Q}^{m+1} \\
\vdots & \ddots & \vdots \\
S_{Q}^{m+1} & \cdots & S_{Q}^{k}
\end{array}\right] \cdot\left[\begin{array}{l}
\Delta Q_{1} \\
\vdots \\
\Delta Q_{m} \\
\Delta Q_{m+1} \\
\vdots \\
\Delta Q_{k}
\end{array}\right]
\end{aligned}
$$

where $\Delta P_{i}$ and $\Delta Q_{i}$ are injected real and reactive power of controllable device; $\Delta P_{m}$ is power adjustment of VSC2 at the DC side, which is equal to the real power adjustment of VSC at the AC side $\Delta P_{m+1} ; \Delta Q_{m+1}$ is the reactive power adjustment of VSC2 at the AC side.

It should be mentioned that the real and reactive power of VSC2 can be independently controlled by setting up the reference values of the real and reactive power, the hybrid AC/DC distribution network is decoupled by VSC2. Thus the sensitivity method can be used for AC and DC parts individually.

\subsection{Priority-based coordination strategy}

Firstly, if the voltage of an AC node is higher than 1.05 p.u., or lower than 0.95 p.u., the reactive power of the DG, $Q_{D G}$, in the same partition is given priority to regulate the voltage when real power hasn't used up all power rating of the DGs. Equation (18) is used to preliminarily calculate the adjustment of $Q_{D G}$ based on the maximum voltage deviation of the measured nodes in $[\Delta V]$.

Secondly, if the node voltage is still higher 1.05 p.u., or lower than 0.95 p.u. when $Q_{D G}$ achieve its maximum value under the power rating limit of the DG, then the ESS or VSC2 in the same partition is considered to regulate the voltage because it is more efficient than other devices and has enough power reserve. For VSC2, the reactive power $Q_{V S C 2}^{A C}$, is firstly considered to regulate the voltage because DC lines are not affected by the reactive power regulation of the AC side. For ESSs, the real power $P_{E S S}$, is firstly considered to regulate the voltage in order to reduce electricity purchase from the network.

Equations (17) and (18) are used to preliminarily calculate the adjustments of real and reactive power based on the maximum voltage deviation of the measured nodes in $[\Delta V]$.

If $Q_{V S C 2}^{A C}$ and $P_{E S S}$ cannot be achieved due to the power rating limit of converters, both real and reactive power will be used to regulate node voltages.

The real and reactive power adjustment of $\mathrm{VSC} 2$ and ESS, $\left(P_{V S C 2}^{A C}, Q_{V S C 2}^{A C}, P_{E S S}, Q_{E S S}\right)$ can be obtained through (20).

$$
\left\{\begin{array}{l}
S_{P} \Delta P+S_{Q} \Delta Q=\Delta V \\
\left(P_{V S C 2}^{A C}+\Delta P\right)^{2}+\left(Q_{V S C 2}^{A C}+\Delta Q\right)^{2}=S_{V S C 2}^{2}
\end{array}\right.
$$

where $\Delta V$ is the maximum voltage deviation of the measured nodes $[\Delta V] ; S_{P}$ and $S_{Q}$ are the corresponding sensitivities of the nodes.

If $S_{P}<S_{Q}$, the absolute value of ESSs or VSC2 real power will be reduced in order to release the ratings of converters for reactive power to regulate node voltages more effectively. In case $S_{P}>S_{Q}$, the ratings of converters are used to meet real power, and the absolute value of reactive power will be reduced if real power is limited by current ratings.

Finally, if the node voltage is still higher 1.05 p.u., or lower than 0.95 p.u. after the adjustments of $P_{V S C 2}^{A C}, Q_{V S C 2}^{A C}$, $P_{E S S}, Q_{E S S}$. The curtailment of DG, $P_{D G}$, in the same partition is considered to regulate node voltages. $P_{D G}$ and $Q_{D G}$ can be calculated through (20).

On the other hand, if a DC node voltage is higher than 1.05 p.u. or lower than 0.95 p.u., the real power of ESSs or VSC2 $\left(P_{E S S}\right.$ or $\left.P_{V S C 2}^{A C}\right)$ in the same partition is used to regulate node voltages based on (17). Especially, the 
absolute value of $Q_{V S C 2}^{A C}$ will be reduced in order to release the rating of $\mathrm{VSC} 2$ for real power to regulate node voltages if real power is limited by current ratings of VSC2, and the $P_{V S C 2}^{A C}$ and $Q_{V S C 2}^{A C}$ can be calculated through (20).

The flowchart of priority-based coordination is shown in Fig. 4.

\section{Case studies}

Case studies were conducted to demonstrate the superiority of proposed priority-based coordinated control strategy based on multi-objective partition considering the equivalence of real power to reactive power.

\subsection{Simulation conditions}

A 38-node hybrid AC/DC medium voltage distribution network embedded with DGs and ESSs, as shown in Fig. 5, is used to test the proposed method. The AC line of the 38-node network, which is denoted by the dashed red line, is modified based on IEEE 33-node distribution network, while the DC line, which is represented by the solid black line, is modified by [26]. The rated power of DGs is 300 $\mathrm{kW}$, and the rated power of ESSs is $500 \mathrm{~kW}$. The rated

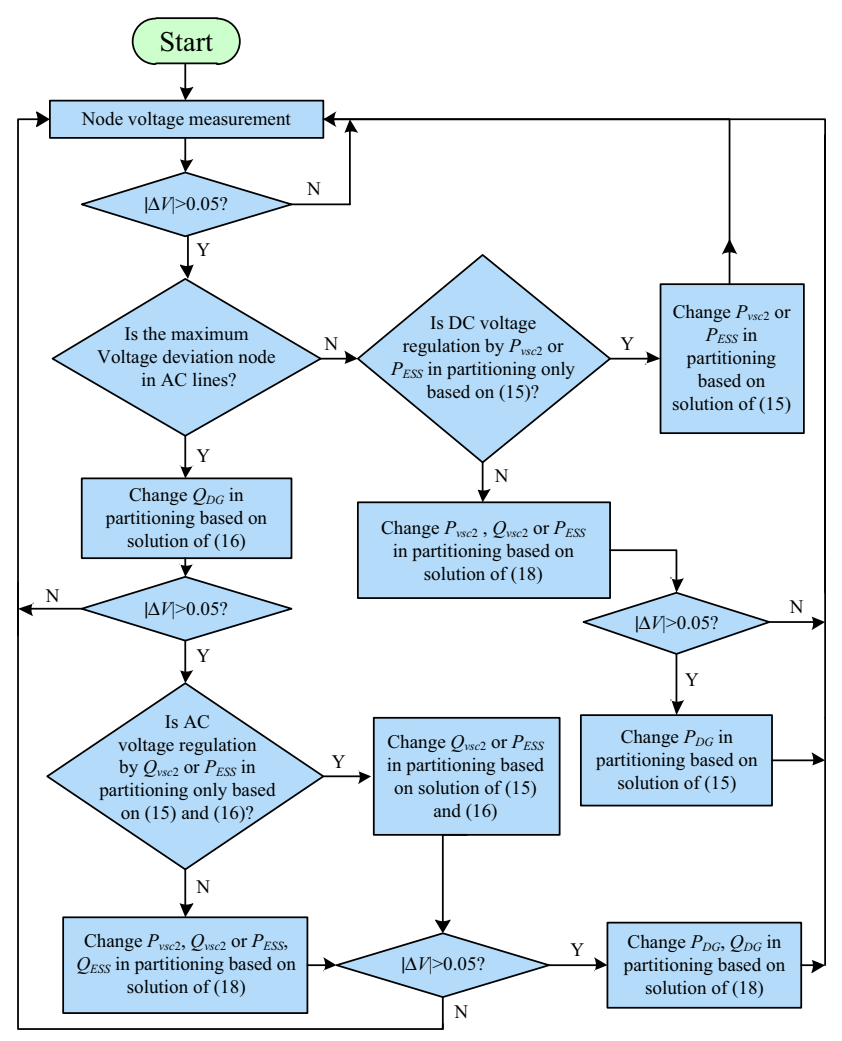

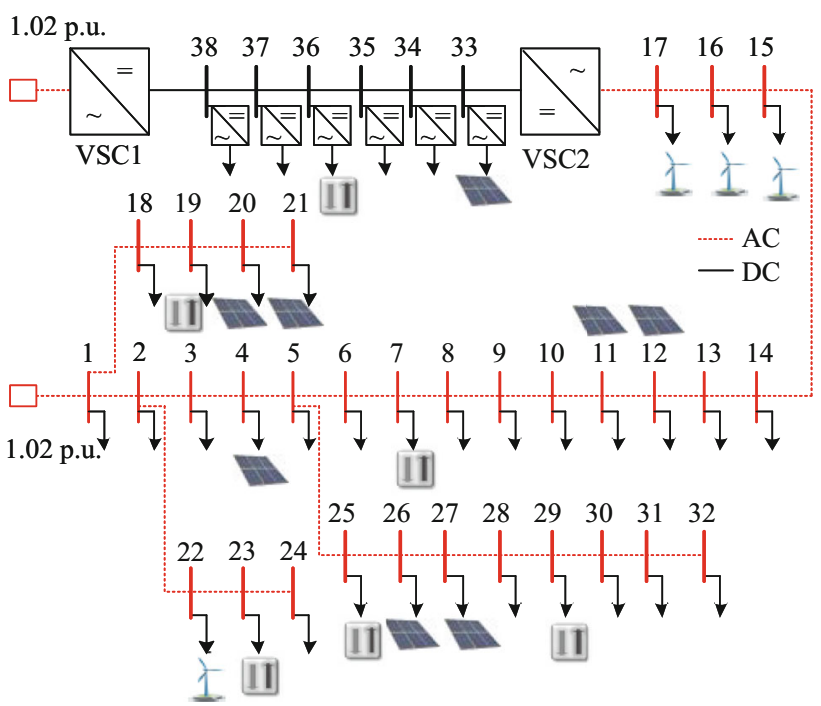

Fig. 5 A 38-node hybrid AC/DC medium voltage distribution network integrated DGs and ESSs

capacity of VSC2 is $3 \mathrm{MW}$. The voltage on the secondary side of the distribution transformers are fixed as 1.02 p.u..

\subsection{Study-1 benefits of considering equivalence of real power to reactive power during partition}

In Case 1 the equivalence of real power to reactive power is considered in partition results, and in Case 2 only the capability of reactive power is considered.

Node 6 belongs to partition 1 in Case 2, as shown in Table 1 , because the regulation capability of partition 1 is larger than partition 2 due to the DGs at node 4, 26, 27, although the electrical distance of node 6 to partition 2 is small. After considering real power capability in power reserve, Node 6 belongs to partition 2 in Case 1, as shown in Fig. 6, because the voltage can be regulated more efficient by the ESS at node 7, which has enough capability.

Node 10,11, 12 belong to partition 2 in Case 2, instead of partition 3 in Case 1. That is because the regulation capacity increment of VSC2 is larger than ESS considering the capability of real power due to large rated capacity of VSC2.

In DC sides, node 35 belongs to partition 8 instead of partition 7 using the proposed method. That is because DC node voltages are not influenced by reactive power, and the real power of VSC2 is limited by both AC and DC lines.

Regulation capacities in Case 1 and Case 2 are nearly the same, as shown in Fig 7, because the SSD of power reserve is used, and the average value of power reserve is different for the two cases. However, the regulation efficiency in Case 1 is better than that in Case 2, because some nodes, e.g. node 6 , belong to the nearest partition after

Fig. 4 Flowchart of proposed priority-based coordination strategy 
Table 1 Partition results of different solution methods

\begin{tabular}{ll}
\hline Solution method & Node number of partition \\
\hline Case 1 & $\{1,2,3,4,5,25,26,27\}\{6,7,8,9\}\{10,11,12,13,14,15,16,17\}\{18,19,20,21\}\{22,23,24\}\{28,29,30,31,32\}\{33,34\}\{35,36,37,38\}$ \\
Case 2 & $\{1,2,3,4,5,6,25,26\}\{7,8,9,10,11,12\}\{13,14,15,16,17\}\{18,19,20,21\}\{22,23,24\}\{27,28,29,30,31,32\}\{33,34,35\}\{36,37,38\}$ \\
\hline
\end{tabular}

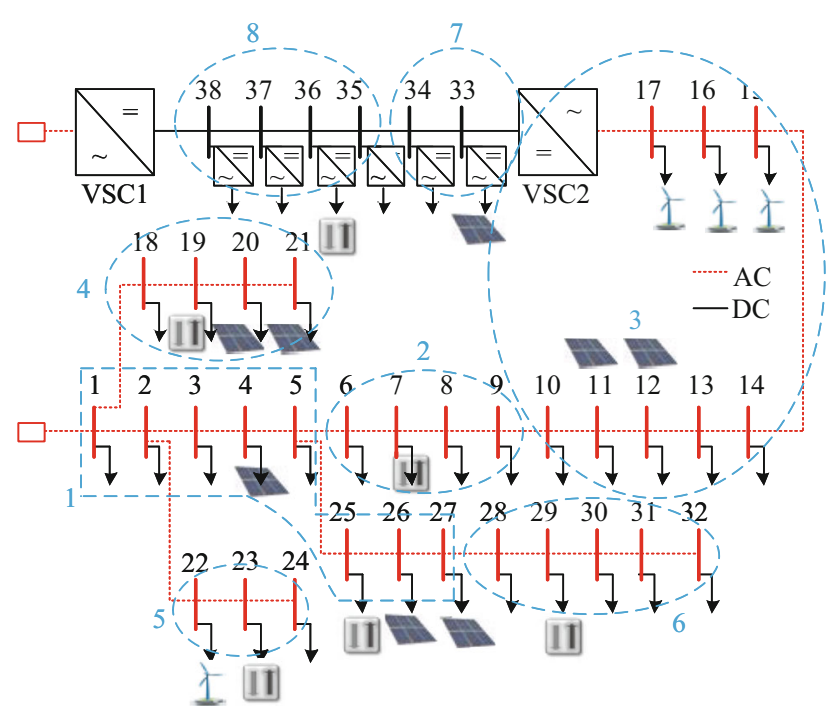

Fig. 6 Partition result of Case 1

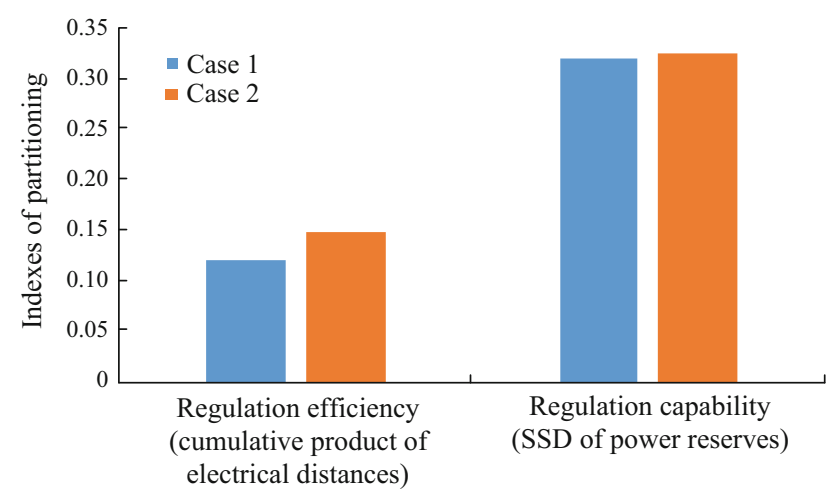

Fig. 7 Partition indexes

considering the equivalence of real power to reactive power.

\subsection{Study-2 benefits of proposed priority-based coordinated control strategy}

Four events of DG and load variation are shown in Fig. 8, during 30-80, 100-180, 200-280, 300-380 s, respectively. All the fluctuations are assumed to be unexpected. Assuming VSC2, DGs, and ESSs can measure node voltage and change power output every 5 seconds. The day-ahead outputs of $P_{V S C 2}^{A C}, Q_{V S C 2}^{A C}, P_{E S S}, Q_{E S S}$ and $Q_{D G}$, as

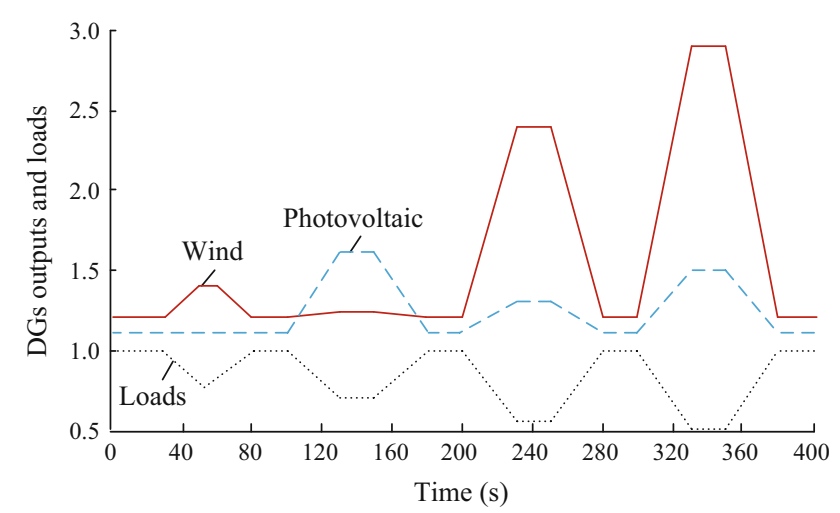

Fig. 8 Loads and DGs outputs of $400 \mathrm{~s}$ in real-time

well as loads and DG real power outputs are assumed to had been determined according to [4, 26].

From $30 \mathrm{~s}$ to $80 \mathrm{~s}$, the DG output increment and load demand reduction as shown in Fig. 8, reactive power of DGs doesn't change until the voltage of node 17 reaches 1.05 p.u. at $50 \mathrm{~s}$, when the proposed priority-based coordinated control strategy is triggered, as shown in Fig. 9. Reactive power outputs of DGs in partition 3 change to $-0.06 \mathrm{MW}$ at $60 \mathrm{~s}$ according to (16), and the voltage of node 17 is regulated to 1.025 p.u., then $Q_{D G}$ come back to the initial values at $70 \mathrm{~s}$.

When the voltage of node 12 is above 1.05 p.u. at $120 \mathrm{~s}$, the reactive power of DGs in partition 3 cannot control the voltage back to 1.03 p.u.. Thus, VSC 2 change its reactive power output during 120-150 s, while in Case 2, the ESS in partition 2 change its real power according to (15).

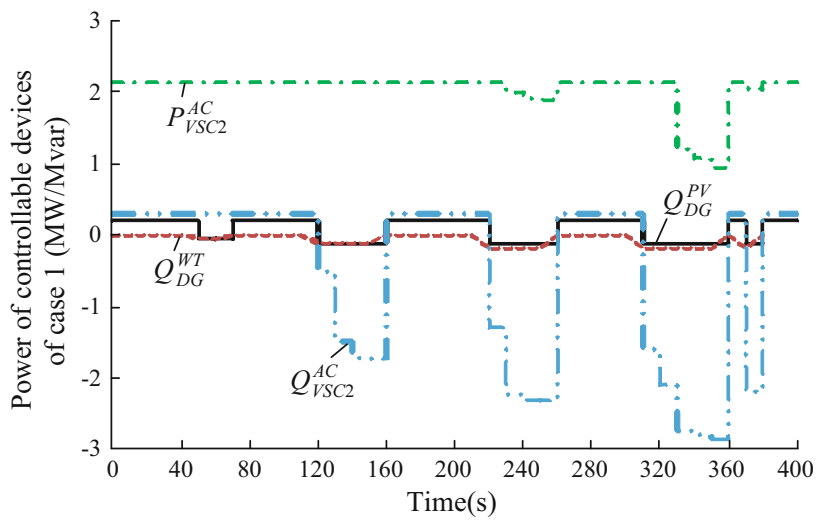

Fig. 9 Power of controllable devices for Case 1 


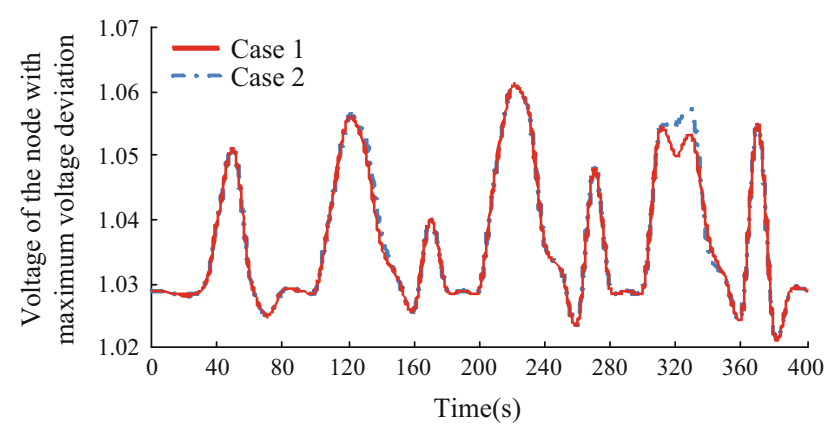

Fig. 10 Voltage deviation

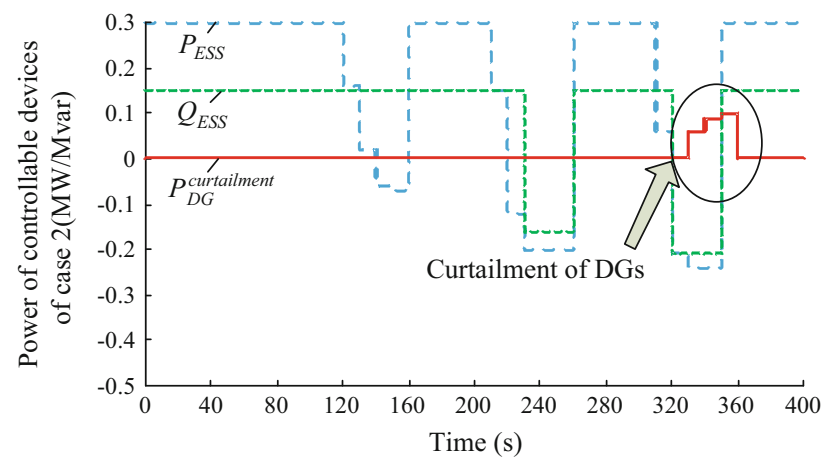

Fig. 11 Power of controllable devices for Case 2

If the DG outputs further increased from 200 to $280 \mathrm{~s}$, $\mathrm{VSC} 2$ reactive power alone is not able to control the voltage of node 17 back to 1.03 p.u., as shown in Fig. 10. Through calculation using (18), VSC2 reduces its real power output, in order to give more capacity for increasing reactive power absorbed from the AC lines. While in Case 2, the ESS in partition 2 reduces its reactive power and increases its real power absorbed from distribution networks.

If the DG and load variations increase significantly, as shown during 300-380 s in Fig. 8, more real power of VSC2 needs to be reduced for reactive power regulation. However, in Case 2, the ESS cannot control the voltage back to 1.03 p.u. due to limited regulation capability, although both real and reactive power are coordinated calculated. Curtailments of DGs happen during 330-350 s, as shown in Fig. 11, which is about $1.4 \mathrm{kWh}$ in total. It can be observed that such incremental accommodations of DG outputs are enabled by regulating node voltages within a security range when unexpected fast fluctuations of DGs and loads happen.

\section{Conclusion}

By considering the equivalence of real power to reactive power in voltage regulation, a new partition method is proposed for hybrid AC/DC medium voltage distribution networks. In real-time, real and reactive power are coordinated to control the voltage in each partition obtained by the proposed method. The electrical distances and the available real and reactive power control capacity are used to optimally partition a hybrid AC/DC medium voltage distribution network. A priority-based control strategy, which coordinates different controllable devices in a partition, is proposed to achieve the voltage regulation while reducing curtailments of renewable energies.

When variations of DGs and loads happen and the reactive power of DGs are not able to regulate the voltages within $[0.97,1.03]$ due to the limited power ratings of the DGs, the VSC between AC and DC lines, or the ESS in the same partition will control node voltages.

Case studies indicate that through the proposed method $1.4 \mathrm{kWh}$ more energy of DGs can be accommodated during $400 \mathrm{~s}$ in a 38-node hybrid AC/DC medium voltage distribution.

Open Access This article is distributed under the terms of the Creative Commons Attribution 4.0 International License (http:// creativecommons.org/licenses/by/4.0/), which permits unrestricted use, distribution, and reproduction in any medium, provided you give appropriate credit to the original author(s) and the source, provide a link to the Creative Commons license, and indicate if changes were made.

\section{References}

[1] Salih SN, Chen P (2016) On coordinated control of OLTC and reactive power compensation for voltage regulation in distribution systems with wind power. IEEE Trans Power Syst 31(5):4026-4035

[2] Kim Y, Ahn S, Hwang P et al (2013) Coordinated control of a DG and voltage control devices using a dynamic programming algorithm. IEEE Trans Power Syst 28(1):42-51

[3] Capitanescu F, Ochoa LF, Margossian H et al (2015) Assessing the potential of network reconfiguration to improve distributed generation hosting capacity in active distribution systems. IEEE Trans Power syst 30(1):346-356

[4] Zhang L, Tang W, Liang J et al (2016) Coordinated day-ahead reactive power dispatch in distribution network based on real power forecast errors. IEEE Trans Power Syst 31(3):2472-2480

[5] Hong YY, Luo YF (2009) Optimal VAR control considering wind farms using probabilistic load-flow and gray-based genetic algorithms. IEEE Trans Power Deliv 24(3):1441-1449

[6] Elnozahy MS, Salama MMA (2015) Uncertainty-based design of a bilayer distribution system for improved integration of PHEVs and PV arrays. IEEE Trans Sust Energ 6(3):1-16

[7] Wang L, Liang DH, Crossland AF et al (2015) Coordination of multiple energy storage units in a low-voltage distribution network. IEEE Trans Smart Grid 6(6):2906-2918

[8] Safdarian A, Fotuhi-Firuzabad M, Lehtonen M (2013) A stochastic framework for short-term operation of a distribution company. IEEE Trans Power Syst 28(4):4712-4721

[9] Huang J, Liu M, Zhang J et al (2015) Analysis and field test on reactive capability of photovoltaic power plants based on clusters of inverters. J Mod Power Syst Clean Energy 5(2):283-289. doi:10.1007/s40565-015-0154-7 
[10] Li XJ, Yao LZ, Hui D (2016) Optimal control and management of a large-scale battery energy storage system to mitigate fluctuation and intermittence of renewable generations. J Mod Power Syst Clean Energy 4(4):593-603. doi:10.1007/s40565016-0247-y

[11] Feng L, Zhang J, Li G et al (2016) Cost reduction of a hybrid energy storage system considering correlation between wind and PV power. Prot Control Mod Power Syst 1(1):11-19

[12] Hu B, Wang H, Yao S (2017) Optimal economic operation of isolated community microgrid incorporating temperature controlling devices. Prot Control Mod Power Syst 2(1):6-16

[13] Zhang L, Tang W, Liang J et al (2016) A medium voltage hybrid ACDC distribution network and its economic analysis. In: The 12th IET international conference on AC and DC power transmission, Beijing, China, 28-29 May, 2016, 6 pp

[14] Zhang L, Tang W, Bai MK et al (2013) Comprehensive configuration of wind turbine and switch capacitor in distribution network based on bi-level programming. In: IEEE-PES APPEEC, Hong Kong, China, 8-11 Dec 2013, 5 pp

[15] Bracale A, Angelino R, Carpinelli G et al (2011) Dispersed generation units providing system ancillary services in distribution networks by a centralised control. IET Renew Power Gener 5(4):311-321

[16] Chaudhary SK, Guerrero JM, Teodorescu R (2015) Enhancing the capacity of the AC distribution system using DC interlinks 2014: a step toward future DC grid. IEEE Trans Smart Grid 6(4):1722-1729

[17] Eajal AA, Shaaban MF, Ponnambalam K et al (2016) Stochastic centralized dispatch scheme for AC/DC hybrid smart distribution systems. IEEE Trans Sust Energ 7(3):1046-1059

[18] Kleinberg MR, Miu K, Segal N et al (2014) A partition method for distributed capacitor control of electric power distribution systems. IEEE Trans Power Syst 29(2):637-644

[19] Mehrjerdi H, Lefebvre S, Saad M et al (2013) A decentralized control of partitioned power networks for voltage regulation and prevention against disturbance propagation. IEEE Trans Power Syst 28(2):1461-1469

[20] Nayeripour M, Fallahzadeh-Abarghouei H, Waffenschmidt E et al (2016) Coordinated online voltage management of distributed generation using network partition. Electr Power Syst Res 141:202-209

[21] Wang P, Liang DH, Yi J et al (2014) Integrating electrical energy storage into coordinated voltage control schemes for distribution networks. IEEE Trans Smart Grid 5:1018-1032

[22] Hu ZC, Wang XF, Wang XL et al (2005) A two-layered network partition approach for optimal reactive power dispatching. Power Syst Technol 24:37-41

[23] Fernández CJC, Martínez FJ, Hervás C et al (2010) Sensitivity versus accuracy in multiclass problems using memetic Pareto evolutionary neural networks. IEEE Trans Neural Netw 21(5):750-770

[24] Zhang LM, Tang W, Liu Y et al (2015) Multiobjective optimization and decision-making for DG planning considering benefits between distribution company and DGs owner. Int $\mathbf{J}$ Electr Power Energy Syst 73:465-474

[25] Brenna M, Berardinis ED, Carpini LD et al (2013) Automatic distributed voltage control algorithm in smart grids applications. IEEE Trans Smart Grid 4(2):877-885
[26] Zhang L, Tang W, Liang J et al (2016) Power-voltage coordinated control in hybrid AC/DC medium voltage distribution networks based on VSC. Proc Chin Soc Electr Eng 36(22):6067-6075

Lu ZHANG received the Ph.D and B.S. degree in College of Information and Electrical Engineering from China Agricultural University, Beijing, China, in 2016 and 2011 . He is currently a postdoctor with Tsinghua University with the Department of Electrical Engineering, State Key Laboratory of Control and Simulation of Power System and Generation Equipment from Tsinghua University. His main research interests include hybrid AC/DC distribution network, renewable energy generation and Active distribution network.

Ying CHEN received the B.E. and Ph.D degrees from Tsinghua University, Beijing, China, in 2001 and 2006, respectively, both in electrical engineering. He is currently an Associate Professor with the Department of Electrical Engineering and Applied Electronic Technology, Tsinghua University. His research interests include parallel and distributed computing, electromagnetic transient simulation, cyber-physical system modeling, and cyber security of smart grid.

Chen SHEN received the B.Sc. and Ph.D degrees in electrical engineering from Tsinghua University, Beijing, China, in 1993 and 1998, respectively. Since 2009, he has been a Professor in the Electrical Engineering Department with Tsinghua University, where he is currently the Director of Power System Research Institute with the Department of Electrical Engineering. His research focuses on power systems analysis and control, including fast modeling and simulation of smart grids, stability analysis of power systems with wind generation, emergency control and risk assessment of power systems, planning, simulation operation, and control for microgrids.

Wei TANG received the B.Sc. degree from Huazhong University of Science and Technology, Wuhan, China, in 1992 and the Ph.D degrees from Harbin Institute of Technology, Harbin, China, in 1998. From 1998 to 2000, she was a post-doctor with Harbin Engineering University. Currently she is a professor at College of Information and Electrical Engineering, China Agricultural University, Beijing, China. Her research interests include Distribution network economic and security operation, Distributed generation and Active distribution network

Jun LIANG received the B.Sc. degree from Huazhong University of Science and Technology, Wuhan, China, in 1992 and the M.Sc. and Ph.D. degrees from China Electric Power Research Institute, Beijing, China, in 1995 and 1998, respectively. From 1998 to 2001, he was a Senior Engineer with China Electric Power Research Institute. From 2001 to 2005, he was a Research Associate at Imperial College, London, U.K. From 2005 to 2007, he was a Senior Lecturer at the University of Glamorgan, Wales, U.K. Currently he is a Reader at the School of Engineering, Cardiff University, Wales, U.K. His research interests include FACTS devices/HVDC, power system stability and control, power electronics, and renewable power generation. 